\begin{tabular}{|c|c|}
\hline Title & $\begin{array}{l}\text { Preval ence, clinical course, and predictive factors of immune checkpoint inhibitor monotherapy-associated hepatitis in } \\
\text { Japan }\end{array}$ \\
\hline Author(s) & $\begin{array}{l}\text { Kitagataya, T akashi; Suda, Goki; Nagashima, Kazunori; Katsurada, Takehiko; Y amamoto, Koji; Kimura, Megumi; } \\
\text { Maehara, O samu; Y amada, Ren; Shigesawa, Taku; Suzuki, Kazuharu; Nakamura, A kihisa; Ohara, Masatsugu; } \\
\text { Umemura, Machiko; Kawagishi, Naoki; Nakai, Masato; Sho, T akuya; Natsuizaka, Mitsuteru; Morikawa, Kenichi; } \\
\text { Ogawa, Koji; Ohnishi, Shunsuke; Komatsu, Y oshito; Hata, Hiroo; Takeuchi, Satoshi; A be, Takashige; Sakakibara } \\
\text { Konishi, Jun; T eshima, Takanori; Homma, A kihiro; Sakamoto, Naoya }\end{array}$ \\
\hline Citation & $\begin{array}{l}\text { Journal of gastroenterology and hepatology, 35(10), 1782-1788 } \\
\text { https://doi.org/10.1111/jgh.15041 }\end{array}$ \\
\hline Issue Date & $2020-10$ \\
\hline Doc URL & http:/hdl.handle.net/2115/82884 \\
\hline Rights & $\begin{array}{l}\text { This is the peer reviewed version of the following article: Kitagataya, T., Suda, G., Nagashima, K., Katsurada, T., } \\
\text { Y amamoto, K., Kimura, M., Maehara, O., Y amada, R., Shigesawa, T., Suzuki, K., Nakamura, A., Ohara, M., Umemura, } \\
\text { M., Kawagi shi, N., Nakai, M., Sho, T., Natsuizaka, M., Morikawa, K., Ogawa, K., Ohnishi, S., Komatsu, Y., Hata, H., } \\
\text { Takeuchi, S., A be, T., Sakakibara- Konishi, J., T eshima, T., Homma, A., and Sakamoto, N. (2020) Preval ence, clinical } \\
\text { course, and predictive factors of immune checkpoint inhibitor monotherapy- associated hepatitis in Japan. Journal of } \\
\text { Gastroenterology and Hepatology, 35: } 1782-1788 ., \text { which has been published in final form at } \\
\text { https://doi.org/10.1111/jgh.15041. This article may be used for non-commercial purposes in accordance with Wiley } \\
\text { Terms and Conditions for U se of Self-A rchived Versions. }\end{array}$ \\
\hline Tyре & article (author version) \\
\hline Additional Information & There are other files related to this item in HUSCAP. Check the above URL. \\
\hline File Information & J Gastroenterol Hepatol 2020 Mar 18 15041.pdf \\
\hline
\end{tabular}

Instructions for use 
1 Prevalence, clinical course, and predictive factors of immune checkpoint inhibitor

4 Abstract

5 Background and Aim: Immune checkpoint inhibitors (ICI) have revolutionized anti6 malignancy therapy and thus have been increasingly used. Although ICI may cause 7 immune-related adverse events (irAE) in various organs, including the liver, the 8 prevalence and predictive factors of irAE have not been clarified.

9 Methods: In this retrospective study, consecutive patients who had malignancies and were treated with ICI without other chemotherapeutic agents at Hokkaido University Hospital between 2014 and 2019 were screened. Patients were excluded if they were $<20$ years old and had insufficient clinical data.

Results: Of the 233 patients screened, 202 patients met the inclusion criteria and were included in the analysis. The patients were aged 25-92 years, and 60.9\% were male. The patients received nivolumab $(n=137)$, pembrolizumab $(n=45)$, ipilimumab $(n=17)$, atezolizumab $(n=2)$, and avelumab $(n=1)$. The prevalence of any grade and grade $\geq 3$ irAE hepatitis was $8.4 \%(17 / 202)$ and 4.0\% (8/202), respectively. irAE hepatitis occurred at a median duration of 42 days in any grade and 36 days in grade $\geq 3$ after ICI initiation. The clinical course of grade $\geq 3$ irAE hepatitis was generally favorable; however, $50 \%$ required corticosteroid treatment and two patients required additional mycophenolate mofetil. Female sex and history of ICI treatment were significantly associated with the incidence of grade $\geq 3$ irAE hepatitis.

Conclusions: Grade $\geq 3$ irAE hepatitis was observed in $4.0 \%$ of the patients who were treated with ICI. Female sex and history of ICI treatment were significantly associated 
1 with the incidence of grade $\geq 3$ irAE hepatitis.

2

3 Keywords: female, hepatitis, immune checkpoint inhibitor, immune system, adverse

4 events, risk factor, irAE

5

6 
1

2 Cancer is the second leading cause of death globally, and approximately 9.6 million

3 people have died of the disease. ${ }^{1}$ Recent advances in the treatment and diagnosis of

4 various cancers have improved patient prognosis. ${ }^{1}$ Among the treatment advances for

5 cancer, the development of immune checkpoint inhibitors (ICI), which target down-

6 regulators of the anti-cancer immune response, is considered an outstanding breakthrough.

7 ICI mainly consist of cytotoxic T-lymphocyte antigen-4 (CTLA-4) inhibitors,

8 programmed cell death protein (PD)-1 inhibitors, and PD ligand inhibitors. Currently, no

9 clinical trial has revealed and confirmed the efficacy of ICI for various malignancies..$^{2-6}$

10 Nevertheless, ICI have durable responses at a relatively high rate, which is not observed with conventional chemotherapy; however, they occasionally cause immune-related adverse events (irAE), which are associated with over-activation of the immune system. ${ }^{7}$ Importantly, irAE could be observed in any organ, including the liver, and may sometimes develop into severe adverse events (AE). Considering the increasing utilization of ICI for various malignancies, evaluating the irAE due to ICI use is crucial, because most clinical trials exclude patients with complications including, renal dysfunction, anemia, thrombocytopenia, and liver dysfunction ${ }^{2,5,6}$, who could be adapted to ICI treatment in the real-world setting.

Although numerous predictive factors of ICI response have been analyzed and reported, data on the predictive factors of irAE are limited. ${ }^{7}$ Currently, the identified predictive factors of the occurrence of irAE include sarcopenia, ${ }^{8}$ female sex, and serum IL-6, ${ }^{9}$ microbiota, ${ }^{10}$ and blood cell counts. ${ }^{11}$ However, real-world data and studies focusing on irAE hepatitis analysis are scarce. Moreover, the use of ICI has been increasing; ICI have been administered to patients with hepatocellular carcinoma, who commonly have liver 
1 dysfunction. Thus, clarifying the prevalence and risk factors of irAE hepatitis is a

2 clinically important issue.

3 Hence, in this study, we aimed to evaluate the prevalence and clinical course of irAE

4 hepatitis in Japanese patients who were treated with ICI and to determine and analyze the 5 predictive factors of irAE hepatitis.

6

7

8 Methods

$9 \quad$ Patients and study design

10 In this retrospective study, consecutive patients who had malignancies and were treated 11 with ICI monotherapy with no combination of other chemotherapeutic agents at 12 Hokkaido University Hospital between August 2014 and July 2019 were screened.

13 Patients were excluded if they were $<20$ years old; were receiving other chemotherapeutic 14 agents, including multikinase inhibitors and cytotoxic agents; and lacked clinical information, such as evaluation of irAE. The obtained data included ICI regimen, sex, age, primary malignancy, existence of metastasis, treatment protocol before ICI treatment, blood cell count, laboratory results, skeletal muscle mass based on psoas muscle mass index (PMI), and hepatitis B infection. Moreover, patients were assessed using laboratory tests and physical findings at least every 2 to 3 weeks.

This study was approved by the ethics committees of Hokkaido University Hospital (clinical research number: IRB 018-0405). The study protocol conformed to the ethical guidelines of the Declaration of Helsinki. Patients provided written informed consent to participate or did not decline to participate this study. 
1

2

3

4

5

6

7

\section{Treatment protocol}

In patients with lung cancer, nivolumab $3 \mathrm{mg} / \mathrm{kg}$ every 2 weeks ( $\mathrm{n}=44$ ), pembrolizumab 200 mg/body every 3 weeks ( $n=32)$, or atezolizumab 1200 mg/body every 3 weeks (n=2) was administered. In patients with melanoma, nivolumab 2 mg/kg every 3 weeks ( $n=38)$, pembrolizumab $2 \mathrm{mg} / \mathrm{kg}$ every 2 weeks (n=7), or ipilimumab $3 \mathrm{mg} / \mathrm{kg}$ every 3 weeks ( $\mathrm{n}=17)$ was administered. Patients with renal cell carcinoma received nivolumab $3 \mathrm{mg} / \mathrm{kg}$ every 2 weeks $(n=24)$ or pembrolizumab 200 mg/body every 3 weeks $(n=4)$, and those with oral carcinoma $(n=6)$, gastric cancer $(n=5)$, or pharyngeal carcinoma $(n=15)$ received nivolumab 3 mg/kg every 2 weeks. In patients with other carcinomas, nivolumab 3 mg/kg or 240 mg/body every 2 weeks ( $n=5)$, pembrolizumab 200 mg/body every 3 weeks ( $n=2)$, or avelumab $10 \mathrm{mg} / \mathrm{kg}$ every 2 weeks ( $\mathrm{n}=1$ ) was administered.

\section{IrAE hepatitis}

All patients with liver dysfunction during and after the ICI treatment were evaluated using the Digestive Disease Week Japan 2004 (DDW-J) scale, which has been proposed as a tool for the diagnosis of drug-induced liver injury. ${ }^{12}$ Based on the scale, a score of $\geq 3$ (which means "possible” and "probable” drug-induced liver injury) indicates irAE hepatitis in this study. In addition, treatment and treatment outcomes of irAE hepatitis were also evaluated.

The irAE hepatitis grade was defined according to National Cancer Institute Common Terminology Criteria for Adverse Events (CTCAE; version 4.0) and American Society of Clinical Oncology Clinical Practice Guideline. ${ }^{13}$ The grading of the severity of ICIinduced hepatitis was as follows: grade 1: AST or ALT 1-3×upper limit of normal (ULN) and/or T-BIL 1-1.5×ULN, grade 2: AST or ALT 3-5×ULN and/or T-BIL 1.5-3×ULN, 


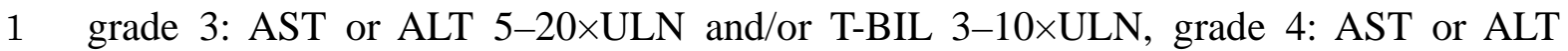

$2 \geq 20 \times \mathrm{ULN}$ and/or T-Bil $\geq 10 \times \mathrm{ULN}$, and grade 5: death. Moreover, we conducted a 3 subgroup analysis considering factors potentially associated with irAE hepatitis grades $4 \quad 1-4$ and grades 3-4.

5

$6 \quad$ Skeletal muscle mass by PMI

7 The relationship between irAE hepatitis and loss of skeletal muscle mass was assessed 8 according to a previous report. ${ }^{8}$ We evaluated skeletal muscle mass using the PMI on 9 computed tomography images. Based on a previous report, PMI was calculated as 10 follows: the sum of the L3 level cross-sectional area of the right and left psoas muscle mass was measured by manual tracing; subsequently, the value was divided by the height squared $\left(\mathrm{cm}^{2} / \mathrm{m}^{2}\right) \cdot{ }^{14,15}$ Loss of skeletal muscle mass was evaluated according to a previous report. ${ }^{16}$

\section{Statistical analysis}

Statistical analysis of categorical data was performed using the chi-square test, and statistical analyses of continuous variables were performed using the Mann-Whitney U test. In this study, a p-value $<0.05$ was considered statistically significant. We performed the statistical analyses with Prism 7.03 (GraphPad Software, Inc., La Jolla, CA).

\section{Results}


1 Baseline characteristics of the patients

2 In this retrospective study, of the 233 patients who had malignancies and were treated

3 with ICI without combination with other chemotherapeutic agents, 202 patients who had

4 sufficient clinical information and data on irAE evaluation were included in this study.

5 Thirty-one patients were excluded because they did not have proper AE evaluation, they

6 had insufficient treatment duration, or they had insufficient clinical information (Fig. 1).

7 The baseline characteristics of the 202 patients treated with ICI are shown in Table 1 . The

8 patients were aged 25-92 years (median, 67 years), and 60.9\% (123/202) were male.

9 Seventy-eight, 62, 28, 15, 6, 5, and 8 patients had lung cancer, melanoma, renal cell

10 carcinoma, pharyngeal cancer, oral cancer, gastric cancer, and other malignancies

11 (malignant lymphoma [n=1], external auditory canal cancer [n=3], cancer of unknown

12 primary [ $\mathrm{n}=1]$, bladder cancer [ $\mathrm{n}=2]$, and Merkel cell carcinoma [n=1]), respectively. A

13 total of $137,45,17,2$, and 1 patient received nivolumab, pembrolizumab, ipilimumab,

14 atezolizumab, and avelumab, respectively. A total of $16(7.9 \%)$ and 6 (3.0\%) patients

15 previously received ICI once and twice, respectively. Of 22 patients with previous ICI, a

16 total of 16 patients discontinued ICI due to disease progression, and 6 patients due to AE.

17 Regarding skeletal muscle loss, we evaluated the PMI. Median PMI values were 4.37

18 (1.47-9.15) in males and 3.03 (1.18-6.89) in females. Laboratory findings and hepatitis

19 B virus infection status are shown in Table 1.

20 IrAE hepatitis prevalence and factors associated with the occurrence of irAE 21 hepatitis

22 As shown in Table 2, 8.4\% (17/202) of the patients (median age, 66 [range, 25-82] years;

23 female, 8 [47\%]) had any grade of irAE hepatitis and 4.0\% (8/202) of the patients (median

24 age, 59 [range, 25-79] years; female, 7 [88\%]) had grade $\geq 3$ irAE hepatitis. In all patients 
1 with irAE hepatitis, the relationship between acute hepatitis and ICI was classified as

2 possible or probable according to the DDW-J 2004 scale. Moreover, acute hepatitis

3 occurred at a median duration of 42 (range, 14-100) days and 36 (range, 14-100) days

4 after ICI initiation in patients with any grade and with grade $\geq 3$ irAE hepatitis, 5 respectively.

6 Subsequently, we analyzed the factors associated with irAE hepatitis. As shown in Table

7 3, among the baseline patient characteristics, administration of CTLA-4 inhibitor and 8 history of ICI treatment were significantly associated with occurrence of any grade of 9 irAE hepatitis. Importantly, female sex and previous ICI treatment were significantly 10 associated with the occurrence of grade $\geq 3 \mathrm{irAE}$ hepatitis. Treatment response and changes in blood cell count did not influence the occurrence of any grade of irAE hepatitis and grade $\geq 3$ irAE hepatitis (Supplementary Table 1). Additionally, any specific relationship between incidence of irAE hepatitis and other irAEs was not observed in this study (Supplementary Table 2).

\section{Clinical features and clinical course of patients with grade $\geq 3$ irAE hepatitis}

Table 4 shows the baseline characteristics and clinical course of eight patients with grade $\geq 3$ irAE hepatitis. Of the eight patients, four were treated with prednisolone (PSL) (1-2 $\mathrm{mg} / \mathrm{kg} /$ day or $1000 \mathrm{mg} /$ day for 3 days and, subsequently, $1 \mathrm{mg} / \mathrm{kg} /$ day). Of the four patients treated with PSL, two patients required additional treatment with mycophenolate mofetil (MMF) after PSL treatment. Four patients did not require corticosteroid therapy and experienced a spontaneous hepatitis improvement. 


\section{Discussion}

2 Immune checkpoint inhibitors have revolutionized anti-malignancy therapy and have

3 been increasingly used; ${ }^{2-6}$ however, they sometimes cause irAE, which could be observed

4 in various organs, including the liver. Currently, irAE prediction has been challenging. ${ }^{7}$

5 In this study, we analyzed the prevalence and the risk factors of irAE hepatitis in patients

6 treated with ICI without any another chemotherapeutic agent. We revealed that the

7 prevalence of any grade and grade $\geq 3$ irAE hepatitis associated with ICI was $8.4 \%$

8 (17/202) and 4.0\% (8/202), respectively. Female sex and previous ICI treatment were

9 significantly associated with the incidence of grade $\geq 3$ irAE hepatitis. The clinical course

10 of irAE hepatitis was generally favorable; however, $50 \%$ of the patients were treated with

11 corticosteroid and 25\% of the patients required additional MMF administration.

12 In this study, given the retrospective nature of study, the treatment approach for patients

13 with grade $\geq 3$ irAE hepatitis was determined by the attending physician or hepatologist

14 referring to published guidelines ${ }^{13}$, package inserts of ICIs, and the patient's condition.

15 More recent patients with severe irAE hepatitis were referred to a hepatologist for

16 consultation; however, early phase cases were treated by the decision of the attending

17 physician. Among patients with grade $\geq 3$ irAE hepatitis in our study, $50 \%$ of the patients

18 had hepatitis improvement without any corticosteroid treatment. De Martin et al. reported

19 that of 16 patients with grade $\geq 3$ irAE hepatitis, 38\% did not receive any corticosteroid

20 therapy and these patients experienced spontaneous improvement, ${ }^{17}$ whereas Riveiro-

21 Barciela et al. ${ }^{18}$ recently reported acute liver failure due to immune-mediated hepatitis.

22 Generally, patients treated with ICI have an active malignancy. Thus, liver transplantation

23 is not performed and adequate treatment is crucial. However, as the reported number of

24 patients with grade $\geq 3$ irAE hepatitis remains limited, those who require corticosteroid 
1 therapy and/or MMF have yet to be clarified. While histological findings may be

2 helpful, ${ }^{17}$ further studies are still warranted.

3 To date, established diagnosis methods for irAE hepatitis have not been well clarified, 4 and whether the DDW-J 2004 scale would be compatible for evaluation or diagnosis of 5 irAE hepatitis also has not been clarified. However, quite recently, Sawada et al., reported 6 that the DDW-J 2004 scale might be useful for diagnosing and determining whether

7 steroid treatment is required in patients with PD-1 inhibitor-associated drug-induced liver 8 injury. ${ }^{19}$ Thus, the DDW-J 2004 may be utilized for diagnosis of irAE hepatitis, however,

9 further investigation is required

10 Most cases of irAE are observed within 3-6 months after the initiation of ICI treatment, ${ }^{20 \text {, }}$ $11{ }^{21}$ and the time to onset of irAE differs between organs. ${ }^{20}$ IrAE hepatitis has been reported to occur at a median of 7.3 weeks $^{20}$ in any grade and 5 weeks ${ }^{17}$ in grade $\geq 3$, after ICI treatment initiation. Similar to a previous report, our study showed that irAE hepatitis occurs at a median duration of 42 (range, 14-100) days in any grade and 36 (range, 14100) days in grade $\geq 3$. However, previous studies have reported that irAE hepatitis is observed firstly almost 1 year after ICI treatment initiation; ${ }^{17,} 20$ thus, careful monitoring is required at any time after the initiation of ICI treatment.

Moreover, the risk of any grade irAE hepatitis was significantly associated with a history of ICI treatment and CTLA-4 administration in this study. Although each ICI could cause irAE, the prevalence and severity of irAE are highest for the CTLA-4 inhibitor (ipilimumab). ${ }^{22}$ Thus, when administering ipilimumab, greater attention is necessary to detect irAE hepatitis.

23 Furthermore, the prevalence of grade $\geq 3$ irAE hepatitis was significantly higher in 24 females than in males in our study. Several reports revealed that the incidence of irAE 
1 and treatment response could be affected by sex. Valpione et al. demonstrated that in

2 melanoma patients who were treated with ipilimumab, female sex was significantly 3 associated with a higher risk of severe irAE. ${ }^{9}$ Female sex is a risk factor for several 4 autoimmune diseases, including autoimmune hepatitis; ${ }^{23}$ thus, sex could influence the 5 incidence of irAE hepatitis. In addition, as in previous reports, ${ }^{24,} 25$ our study showed that 6 male sex is significantly associated with a high objective response rate (Data not shown).

7 The effect of sex on the occurrence of irAE and ICI treatment response could be attributed 8 to differences in hormones, which could affect immune modulation. Nonetheless, further $9 \quad$ studies are needed.

10 Previous ICI treatment was also significantly associated with the incidence of grade $\geq 3$

11 in this study. However, the reason has not been clearly established, although several hypotheses have been proposed. ICI administration may activate the immune system,

13 resulting in an enhanced immune response. In a previous study, ${ }^{26}$ sequential

14 administration of nivolumab and ipilimumab showed that the increase in ALT was

15 higher in the latter than in the former. Additionally, irAE may occur after completion of

16 ICI treatment; thus, irAE during the succeeding ICI treatment could be due to the

17 initially administered ICI. Riveiro-Barciela et al. reported fulminant hepatitis due to the second ICI (CTLA-4 inhibitor) administration. ${ }^{18}$ The patient was initially treated with

19 nivolumab for melanoma and developed grade 2 hepatitis, which was treated with a

20 corticosteroid. When the primary disease progressed, a CTLA-4 inhibitor was

21 administered, and the patients developed fulminant hepatitis thereafter. Thus, irAE hepatitis should be more carefully monitored during retreatment with ICI, especially in patients who developed irAE hepatitis after the initial ICI treatment.

24 Recently, Daly et al., reported that patients with low skeletal muscle mass are more 
1 likely to experience severe treatment-related toxicity to ICI. ${ }^{8}$ Therefore, in this study, we

2 investigated the relationship between low skeletal muscle mass and occurrence of irAE

3 hepatitis. However, as shown in the Table 3, low skeletal muscle mass was not

4 associated with occurrence of irAE hepatitis. In the report by Daly et al., severe

5 hepatitis was not observed, thus, the results from the present study might be consistent

6 with those of Daly et al. To validate the results, further investigation is required.

7 This study has some key strengths as it addressed not only the incidence of irAE

8 hepatitis, but also the clinical course, treatment efficacy, and predictive factors of irAE

9 hepatitis which have not been clearly defined. In addition, because this study was

10 conducted in a real-world setting, patients, who were not included in clinical trials, such

11 as patients with complications, including renal dysfunction, anemia, and

12 thrombocytopenia, were included.

13 This study has also several limitations. First, this was a retrospective single center study,

14 and the included patients were heterogeneous. Second, the number of included patients were limited, especially those with grade $\geq 3$ irAE hepatitis. Third, the treatment regimens for patients with grade $\geq 3$ irAE hepatitis were not decided by a hepatologist in some cases. Lastly, several clinical data were lacking, including liver biopsy, which could be attributed to the retrospective nature of the study. Therefore, prospective studies including a larger patient sample are required to validate our results.

20 In conclusion, the prevalence of any grade and grade $\geq 3$ irAE hepatitis in patients treated with ICI was $8.4 \%$ (17/202) and 4.0\% (8/202), respectively. The clinical course of grade $\geq 3$ irAE hepatitis was generally favorable; however, two (25\%) patients required additional MMF even after corticosteroid administration. Female sex and previous ICI treatment were significantly associated with the incidence of grade $\geq 3$ irAE hepatitis. 
1 Thus, patients with these factors should be carefully monitored for the development of 2 irAE hepatitis, especially when ICI would be administered.

3

4 
1

2

3 [1] Bray F, Ferlay J, Soerjomataram I, Siegel RL, Torre LA, Jemal A. Global cancer

\section{References} statistics 2018: GLOBOCAN estimates of incidence and mortality worldwide for 36 cancers in 185 countries. CA Cancer J Clin. 2018; 68: 394-424.

[2] Robert C, Long GV, Brady B, et al. Nivolumab in previously untreated melanoma without BRAF mutation. N Engl J Med. 2015; 372: 320-30.
[3] Mazza C, Escudier B, Albiges L. Nivolumab in renal cell carcinoma: latest evidence and clinical potential. Ther Adv Med Oncol. 2017; 9: 171-81.

[4] Fehrenbacher L, Spira A, Ballinger M, et al. Atezolizumab versus docetaxel for patients with previously treated non-small-cell lung cancer (POPLAR): a multicentre, openlabel, phase 2 randomised controlled trial. Lancet. 2016; 387: 1837-46.
[5] Gandhi L, Rodriguez-Abreu D, Gadgeel S, et al. Pembrolizumab plus

Chemotherapy in Metastatic Non-Small-Cell Lung Cancer. N Engl J Med. 2018; 378: 207892.

[6] Motzer RJ, Tannir NM, McDermott DF, et al. Nivolumab plus Ipilimumab versus Sunitinib in Advanced Renal-Cell Carcinoma. N Engl J Med. 2018; 378: 1277-90.

\section{[7] Nakamura Y. Biomarkers for Immune Checkpoint Inhibitor-Mediated Tumor} Response and Adverse Events. Front Med (Lausanne). 2019; 6: 119.

[8] Daly LE, Power DG, O'Reilly A, et al. The impact of body composition parameters on ipilimumab toxicity and survival in patients with metastatic melanoma. Br J Cancer. 2017; 116: 310-7.

[9] Valpione S, Pasquali S, Campana LG, et al. Sex and interleukin-6 are prognostic factors for autoimmune toxicity following treatment with anti-CTLA4 blockade. $J$ Transl Med. 2018; 16: 94.

6 [10] Chaput N, Lepage P, Coutzac C, et al. Baseline gut microbiota predicts clinical 7 response and colitis in metastatic melanoma patients treated with ipilimumab. Ann Oncol. 2017; 28: 1368-79.

9 [11] Nakamura Y, Tanaka R, Maruyama H, et al. Correlation between blood cell count and outcome of melanoma patients treated with anti-PD-1 antibodies. Jpn J Clin Oncol. 1 2019; 49: 431-7.

2 [12] Tsutsui A, Nakanuma Y, Takaguchi K, et al. Comparison of Liver Biopsy Findings 3 with the Digestive Disease Week Japan 2004 Scale for Diagnosis of Drug-Induced Liver 4 Injury. Mediators Inflamm. 2015; 2015: 913793.

35 [13] Brahmer JR, Lacchetti C, Schneider BJ, et al. Management of Immune-Related 
1 Adverse Events in Patients Treated With Immune Checkpoint Inhibitor Therapy: American

2 Society of Clinical Oncology Clinical Practice Guideline. J Clin Oncol. 2018; 36: 1714-68.

3 [14] Hamaguchi Y, Kaido T, Okumura S, et al. Proposal for new diagnostic criteria for

4 low skeletal muscle mass based on computed tomography imaging in Asian adults.

$5 \quad$ Nutrition. 2016; 32: 1200-5.

6 [15] Ohara M, Ogawa K, Suda G, et al. L-Carnitine Suppresses Loss of Skeletal Muscle

7 Mass in Patients With Liver Cirrhosis. Hepatol Commun. 2018; 2: 906-18.

8 [16] Hiraoka A, Aibiki T, Okudaira T, et al. Muscle atrophy as pre-sarcopenia in

9 Japanese patients with chronic liver disease: computed tomography is useful for evaluation.

10 J Gastroenterol. 2015; 50: 1206-13.

11 [17] De Martin E, Michot JM, Papouin B, et al. Characterization of liver injury induced 12 by cancer immunotherapy using immune checkpoint inhibitors. $J$ Hepatol. 2018; 68: 11811390.

14 [18] Riveiro-Barciela M, Munoz-Couselo E, Fernandez-Sojo J, Diaz-Mejia N, Parra-

15 Lopez R, Buti M. Acute liver failure due to immune-mediated hepatitis successfully

16 managed with plasma exchange: New settings call for new treatment strategies? J Hepatol. 17 2019; 70: 564-6.

18 [19] Sawada K, Hayashi H, Nakajima S, Hasebe T, Fujiya M, Okumura T. Non-

19 alcoholic fatty liver disease is a potential risk factor for liver injury caused by immune

20 checkpoint inhibitor. J Gastroenterol Hepatol. 2019.

21 [20] Weber JS, Hodi FS, Wolchok JD, et al. Safety Profile of Nivolumab Monotherapy:

22 A Pooled Analysis of Patients With Advanced Melanoma. J Clin Oncol. 2017; 35: 785-92.

23 [21] Weber JS, Dummer R, de Pril V, Lebbe C, Hodi FS, Investigators MDX. Patterns of onset and resolution of immune-related adverse events of special interest with ipilimumab: detailed safety analysis from a phase 3 trial in patients with advanced melanoma. Cancer. 2013; 119: 1675-82.

27 [22] De Velasco G, Je Y, Bosse D, et al. Comprehensive Meta-analysis of Key ImmuneRelated Adverse Events from CTLA-4 and PD-1/PD-L1 Inhibitors in Cancer Patients. Cancer Immunol Res. 2017; 5: 312-8.

30 [23] Lv T, Li M, Zeng N, et al. Systematic review and meta-analysis on the incidence 31 and prevalence of autoimmune hepatitis in Asian, European, and American population. $J$ 32 Gastroenterol Hepatol. 2019; 34: 1676-84.

33 [24] Wu Y, Ju Q, Jia K, et al. Correlation between sex and efficacy of immune 34 checkpoint inhibitors (PD-1 and CTLA-4 inhibitors). Int J Cancer. 2018; 143: 45-51.

35 [25] Nosrati A, Tsai KK, Goldinger SM, et al. Evaluation of clinicopathological factors 36 in PD-1 response: derivation and validation of a prediction scale for response to PD-1 
1 monotherapy. Br J Cancer. 2017; 116: 1141-7.

2 [26] Weber JS, Gibney G, Sullivan RJ, et al. Sequential administration of nivolumab 3 and ipilimumab with a planned switch in patients with advanced melanoma (CheckMate 4 064): an open-label, randomised, phase 2 trial. Lancet Oncol. 2016; 17: 943-55. 


\section{Figure 1 Study flow}

Patients with carcinoma treated with ICI between August 2014 and July 2019

$$
(n=233)
$$

31 were excluded because of insufficient clinical data or treatment duration.

202 patients were included in the analysis 
Table 1. Baseline characteristics of the patients

$\begin{array}{ll}\text { Total number } & 202\end{array}$

\begin{tabular}{|c|c|}
\hline Age (years) ${ }^{\dagger}$ & $67(25-92)$ \\
\hline Sex (male/female) & $123 / 79$ \\
\hline Primary malignancy (lung/melanoma/kidney/pharyngeal/oral/stomach/others) & $78 / 62 / 28 / 15 / 6 / 5 / 8$ \\
\hline ICI (nivolumab/pembrolizumab/ipilimumab/atezolizumab/avelumab) & $137 / 45 / 17 / 2 / 1$ \\
\hline History of ICI treatment $(0 / 1 / 2)$ & $180 / 16 / 6$ \\
\hline Treatment response (CR/PR/SD/PD/NE) & 2/39/53/104/4 \\
\hline Observation period (day) ${ }^{\dagger}$ & $388(3-1405)$ \\
\hline HBV status (negative/past infection/carrier) & $125 / 74 / 3$ \\
\hline $\mathrm{PMI}^{\dagger}$ & $3.83(1.18-9.15)$ \\
\hline Male $^{\dagger}$ & $4.37(1.47-9.154)$ \\
\hline Female $^{\dagger}$ & $3.03(1.18-6.89)$ \\
\hline Low PMI (\%) & 19.3 \\
\hline Baseline white blood cell count $^{\dagger}$ & $5700(2700-25200)$ \\
\hline Baseline red blood cell count $\left(\times 10^{4}\right)^{\dagger}$ & $390(178-530)$ \\
\hline Baseline platelet count $\left(\times 10^{3}\right)^{\dagger}$ & $25.4(5.5-75.1)$ \\
\hline Baseline TP $(\mathrm{g} / \mathrm{dL})^{\dagger}$ & $6.75(3.9-8.8)$ \\
\hline Baseline Alb (g/dL) ${ }^{\dagger}$ & $3.7(1.4-5.1)$ \\
\hline Baseline total bilirubin (mg/dL) ${ }^{\dagger}$ & $0.55(0.2-4.8)$ \\
\hline Baseline AST level (IU/L) ${ }^{\dagger}$ & $22(8-96)$ \\
\hline Baseline ALT level (IU/L) ${ }^{\dagger}$ & $15.5(5-92)$ \\
\hline Baseline LDH (IU/L) ${ }^{\dagger}$ & $213.5(44-3129)$ \\
\hline Baseline ALP (IU/mL) ${ }^{\dagger}$ & $246(91-2393)$ \\
\hline Baseline $\gamma$-GTP $(\mathrm{IU} / \mathrm{L})^{\dagger}$ & $28(6-700)$ \\
\hline Baseline BUN (mg/dL) ${ }^{\dagger}$ & $15(4-61)$ \\
\hline Baseline eGFR $\left(\mathrm{mL} / \mathrm{min} / 1.73 \mathrm{~m}^{2}\right)^{\dagger}$ & $69.75(3.62-135.5)$ \\
\hline Baseline CRP (mg/dL) ${ }^{\dagger}$ & $0.39(0.02-19.8)$ \\
\hline
\end{tabular}

ICI: immune checkpoint inhibitor, PMI: psoas muscle mass index, CR: complete response, PR: partial response, SD: stable disease, PD: progressive disease: TP: total protein, Alb: albumin, AST: aspartate aminotransferase, ALT: alanine transaminase, LDH: lactate dehydrogenase, ALP: alkaline phosphatase, $\gamma$-GTP: $\gamma$ glutamyl transpeptidase, CK: creatine kinase, BUN: blood urea nitrogen, eGFR: estimated glomerular filtration rate, CRP: C-reactive protein, HBV: hepatitis B virus, NE: not evaluated

${ }^{\dagger}$ Data are shown as median (range) values. 
Table 2 Incidence and characteristics of immune checkpoint inhibitor-associated hepatitis

\begin{tabular}{|c|c|c|c|c|c|c|c|c|c|c|c|c|c|c|}
\hline \multirow{2}{*}{$\begin{array}{c}\text { IrAE } \\
\text { hepatitis } \\
\text { grade }\end{array}$} & \multirow{2}{*}{$\begin{array}{c}\text { Prevalence, } \\
\%\end{array}$} & \multirow{2}{*}{$\begin{array}{l}\text { Age, } \\
\text { years }^{\dagger}\end{array}$} & \multirow{2}{*}{$\begin{array}{c}\text { Sex } \\
(\mathrm{M} / \mathrm{F})\end{array}$} & \multirow{2}{*}{$\begin{array}{c}\text { ICI } \\
\text { (nivo/pem/ipi// } \\
\text { atezo/avelu) }\end{array}$} & \multirow{2}{*}{$\begin{array}{c}\text { History of ICI } \\
(0 / 1 / 2)\end{array}$} & \multirow{2}{*}{$\begin{array}{c}\text { Response } \\
\text { (CR/PR/SD/ } \\
\text { PD/NE) }\end{array}$} & \multicolumn{3}{|c|}{ Baseline } & \multicolumn{5}{|c|}{ Status of hepatitis } \\
\hline & & & & & & & $\begin{array}{c}\mathrm{AST}^{\dagger} \\
(\mathrm{IU} / \mathbf{L})\end{array}$ & $\begin{array}{l}\mathrm{ALT}^{\dagger} \\
\text { (IU/L) }\end{array}$ & $\begin{array}{c}\text { HBV status } \\
\text { (negative/past } \\
\text { infection/carrier) }\end{array}$ & $\begin{array}{l}\mathrm{AST}^{\dagger} \\
\text { (IU/L) }\end{array}$ & $\begin{array}{c}\mathrm{ALT}^{\dagger} \\
\text { (IU/L) }\end{array}$ & $\begin{array}{c}\text { Grade } \\
(1 / 2 / 3 / 4)\end{array}$ & $\begin{array}{c}\text { DDW-J } \\
\text { score }\end{array}$ & Onset of hepatitis, days ${ }^{\dagger}$ \\
\hline Any grade & $\begin{array}{c}8.4 \\
(17 / 202)\end{array}$ & $\begin{array}{c}66 \\
(25-82)\end{array}$ & $9 / 8$ & $8 / 5 / 4 / 0 / 0$ & $12 / 4 / 1$ & $0 / 2 / 4 / 11 / 0$ & $\begin{array}{c}24 \\
(11-51)\end{array}$ & $\begin{array}{c}15 \\
(5-92)\end{array}$ & 8/9/0 & $\begin{array}{c}166 \\
(21-2591)\end{array}$ & $\begin{array}{c}185.5 \\
(61-2488)\end{array}$ & $3 / 6 / 6 / 2$ & $4(3-6)$ & $42(14-100)$ \\
\hline G3/4 & $\begin{array}{c}4.0 \\
(8 / 202)\end{array}$ & $\begin{array}{c}59 \\
(25-79)\end{array}$ & $1 / 7$ & $4 / 2 / 2 / 0 / 0$ & $5 / 2 / 1$ & $0 / \mathbf{1} / \mathbf{1} / \mathbf{6} / \mathbf{0}$ & $\begin{array}{c}23.5 \\
(16-36)\end{array}$ & $\begin{array}{c}17 \\
(9-76)\end{array}$ & $3 / 5 / 0$ & $\begin{array}{c}373 \\
(181-2591)\end{array}$ & $\begin{array}{c}329 \\
(196-2488)\end{array}$ & $0 / 0 / 6 / 2$ & $4(3-6)$ & $35.5(14-100)$ \\
\hline
\end{tabular}

ICI: immune checkpoint inhibitor, nivo: nivolumab, pem: pembrolizumab, ipi: ipilimumab, atezo: atezolizumab, avelu:avelumab, CR: complete response, PR: partial response, SD: stable disease, PD: progressive disease, DDW-J: Digestive Disease Week Japan, AST: aspartate aminotransferase, ALT: alanine transaminase, HBV: hepatitis B virus, NE: not evaluated

${ }^{\dagger}$ Data are shown as median (range) values. 
Table 3. Factors associated with the incidence of immune checkpoint inhibitor-associated hepatitis

\begin{tabular}{|c|c|c|c|c|c|}
\hline & Non-hepatitis & Hepatitis (all grade) & Hepatitis (G3/4) & $\mathrm{p}$ value & $\mathrm{p}$ value \\
\hline Total number (\%) & 185 (91.6\%) & $17(8.4 \%)$ & $8(4.0 \%)$ & Non vs. all & Non vs. G3/4 \\
\hline Age (years) ${ }^{\dagger}$ & $67(29-92)$ & $66(25-82)$ & $59(25-79)$ & n.s. & n.s. \\
\hline Sex (male/female) & $114 / 71$ & $9 / 8$ & $1 / 7$ & n.s. & 0.0094 \\
\hline ICI (nivo/pem/ipi/atezo/avelu) & $129 / 40 / 13 / 2 / 1$ & $8 / 5 / 4 / 0 / 0$ & $4 / 2 / 2 / 0 / 0$ & n.s. & n.s. \\
\hline PD-1 and PD-L1/CTLA4 & $172 / 13$ & $13 / 4$ & $6 / 2$ & 0.0414 & n.s. \\
\hline History of ICI treatment $(0 / 1 / 2)$ & $169 / 12 / 4$ & $12 / 4 / 1$ & $5 / 2 / 1$ & 0.0256 & 0.0236 \\
\hline Treatment response (CR/PR/SD/PD/NE) & $2 / 37 / 49 / 93 / 4$ & $0 / 2 / 4 / 11 / 0$ & $0 / 1 / 1 / 6 / 0$ & n.s. & n.s. \\
\hline Observation period (day) & $385(3-1405)$ & $420(78-1259)$ & $310.5(94-1259)$ & n.s. & n.s. \\
\hline HBV status (negative/past infection/carrier) & $119 / 62 / 4$ & $8 / 9 / 0$ & $3 / 5 / 0$ & n.s. & n.s. \\
\hline Baseline white blood cell count ${ }^{\dagger}$ & $5600(2600-25200)$ & $6600(4100-12200)$ & $6600(4100-12200)$ & n.s. & n.s, \\
\hline Baseline red blood cell count $\left(\times 10^{4}\right)^{\dagger}$ & $390(178-530)$ & $393(337-470)$ & $407.5(350-460)$ & n.s. & n.s. \\
\hline Baseline platelet count $\left(\times 10^{3}\right)^{\dagger}$ & $25.15(5.5-75.1)$ & $28.1(16.7-49.3)$ & $34.75(16.7-49.3)$ & n.s. & n.s. \\
\hline Baseline TP $(\mathrm{g} / \mathrm{dL})^{\dagger}$ & $6.8(3.9-8.8)$ & $6.7(5.4-7.7)$ & $7.0(5.4-7.7)$ & n.s. & n.s. \\
\hline Baseline Alb (g/dL) ${ }^{\dagger}$ & $3.7(1.4-5.1)$ & $3.6(2.9-4.5)$ & $4.05(3.4-4.5)$ & n.s. & n.s. \\
\hline Baseline total bilirubin $(\mathrm{mg} / \mathrm{dL})^{\dagger}$ & $0.6(0.2-4.8)$ & $0.5(0.3-1.6)$ & $0.5(0.3-1.6)$ & n.s. & n.s. \\
\hline Baseline AST level (IU/L) ${ }^{\dagger}$ & $22(8-96)$ & $24(11-51)$ & $23.5(16-36)$ & n.s. & n.s. \\
\hline Baseline ALT level (IU/L) ${ }^{\dagger}$ & $15(5-92)$ & $17(9-76)$ & $16.5(12-49)$ & n.s. & n.s. \\
\hline Baseline LDH (IU/L) ${ }^{\dagger}$ & $212(44-3129)$ & $232(152-369)$ & $233(169-328)$ & n.s. & n.s. \\
\hline Baseline ALP (IU/mL) ${ }^{\dagger}$ & $247(91-2393)$ & $238(111-546)$ & $250(136-328)$ & n.s. & n.s. \\
\hline Baseline $\gamma$-GTP $(\mathrm{IU} / \mathrm{L})^{\dagger}$ & $28(6-700)$ & $28(14-110)$ & $23(14-96)$ & n.s. & n.s. \\
\hline Baseline BUN (mg/dL) ${ }^{\dagger}$ & $15(4-61)$ & $14(10-22)$ & $15(10-22)$ & n.s. & n.s. \\
\hline Baseline eGFR (mL/min/1.73 m²) & $67.6(3.6-135.5)$ & $77.4(54.9-110.3)$ & $81.4(59.0-110.3)$ & n.s. & n.s. \\
\hline Baseline CRP (mg/dL) ${ }^{\dagger}$ & $0.04(<0.02-19.8)$ & $0.16(0.02-11.16)$ & $0.16(0.04-1.54)$ & n.s. & n.s. \\
\hline $\mathrm{PMI}^{\dagger}$ & $3.83(1.18-9.15)$ & $4.56(2.57-7.21)$ & $2.90(2.57-7.21)$ & n.s. & n.s. \\
\hline Low PMI(\%) & 20.54 & 5.88 & 0 & n.s. & n.s. \\
\hline Baseline neutrophil count ${ }^{\dagger}$ & $3698(1574-19685)$ & $4593(2353-10521)$ & 3861 (2353-10521) & n.s. & n.s. \\
\hline Baseline lymphocyte count $^{\dagger}$ & $1179(164-3456)$ & $1344(432-2268)$ & 1365 (974-1756) & n.s. & n.s. \\
\hline Baseline NLR ${ }^{\dagger}$ & $3.32(0.86-29.3)$ & $3.61(1.81-10.8)$ & $2.72(1.86-10.8)$ & n.s. & n.s. \\
\hline Baseline eosinophil count $^{\dagger}$ & $130(0-1163)$ & $138(0-1122)$ & $181(0-1122)$ & n.s. & n.s. \\
\hline
\end{tabular}

ICI: immune checkpoint inhibitor, PMI: psoas muscle mass index, CR: complete response, PR: partial response, SD: stable disease, PD: progressive disease, TP: total protein, Alb: albumin, AST: aspartate aminotransferase, ALT: alanine transaminase, LDH: lactate dehydrogenase, ALP: alkaline phosphatase, $\gamma$-GTP: $\gamma$ glutamyl transpeptidase, CK: creatine kinase, BUN: blood urea nitrogen, eGFR: estimated glomerular filtration rate, CRP: C-reactive protein, HBV: hepatitis B virus, NE: not evaluated, n.s.: not significant

${ }^{\dagger}$ Data are shown as median (range) values. 
Table 4. Principal characteristics of eight patients with grade $\geq 3$ irAE hepatitis

\begin{tabular}{|c|c|c|c|c|c|c|c|c|c|c|c|c|c|c|}
\hline & & & & & & & Baseline & & & & IrAI & hepatitis & $3 / 4)$ & \\
\hline & Age & Sex & $\begin{array}{c}\text { Primary } \\
\text { malignancy }\end{array}$ & ICI & Response & $\begin{array}{c}\text { AST } \\
\text { (IU/L) }\end{array}$ & $\begin{array}{c}\text { ALT } \\
\text { (IU/L) }\end{array}$ & $\begin{array}{l}\mathrm{HBV} \\
\text { status }\end{array}$ & $\begin{array}{l}\text { AST } \\
(\mathrm{IU} / \mathrm{L})\end{array}$ & $\begin{array}{l}\text { ALT } \\
(\mathrm{IU} / \mathrm{L})\end{array}$ & Grade & $\begin{array}{l}\text { DDW-J } \\
\text { score }\end{array}$ & $\begin{array}{c}\text { Onset of } \\
\text { hepatitis } \\
\text { (days) }\end{array}$ & $\begin{array}{c}\text { Treatment for irAE } \\
\text { hepatitis }\end{array}$ \\
\hline 1 & 47 & $\mathrm{~F}$ & Melanoma & ipilimumab & $\mathrm{PD}$ & 23 & 15 & $\begin{array}{c}\text { Past } \\
\text { infection }\end{array}$ & 448 & 581 & G3 & 6 & 42 & SNMC \\
\hline 2 & 47 & F & Melanoma & nivolumab & PD & 25 & 16 & $\begin{array}{c}\text { Past } \\
\text { infection }\end{array}$ & 181 & 207 & G3 & 5 & 24 & - \\
\hline 3 & 79 & $\mathrm{~F}$ & Lung cancer & pembrolizumab & $\mathrm{PD}$ & 16 & 12 & negative & 207 & 196 & G3 & 4 & 40 & UDCA \\
\hline 4 & 52 & $\mathrm{~F}$ & Melanoma & ipilimumab & SD & 29 & 26 & $\begin{array}{c}\text { Past } \\
\text { infection }\end{array}$ & 2591 & 2488 & G4 & 5 & 15 & $\begin{array}{c}\text { PSL } 2 \text { mg/kg/day } \\
\text { +MMF }\end{array}$ \\
\hline 5 & 78 & $\mathrm{~F}$ & Melanoma & nivolumab & $\mathrm{PD}$ & 18 & 14 & negative & 623 & 391 & G3 & 4 & 31 & UDCA \\
\hline 6 & 25 & $\mathrm{M}$ & Lymphoma & nivolumab & PD & 24 & 17 & negative & 583 & 895 & G4 & 4 & 100 & PSL $1000 \mathrm{mg}$ \\
\hline 7 & 66 & $\mathrm{~F}$ & Melanoma & nivolumab & $\mathrm{PD}$ & 18 & 17 & negative & 221 & 235 & G3 & 4 & 14 & PSL 1 mg/kg/day \\
\hline 8 & 68 & $\mathrm{~F}$ & Other & nivolumab & PR & 36 & 49 & $\begin{array}{c}\text { Past } \\
\text { infection }\end{array}$ & 298 & 267 & G3 & 3 & 52 & $\begin{array}{c}\text { PSL } 2 \text { mg/kg/day } \\
+ \text { MMF }\end{array}$ \\
\hline
\end{tabular}

irAE: immune checkpoint inhibitor-related adverse events, ICI: immune checkpoint inhibitor, , CR: complete response, PR: partial response, SD: stable disease, PD: progressive disease, DDW-J: Digestive Disease Week Japan, AST: aspartate aminotransferase, ALT: alanine transaminase, UDCA: ursodeoxycholic acid, PSL: prednisolone, MMF: mycophenolate mofetil, HBV: hepatitis B virus, SNMC: stronger neominophagen $\mathrm{C}$

Data are shown as median (range) values. 